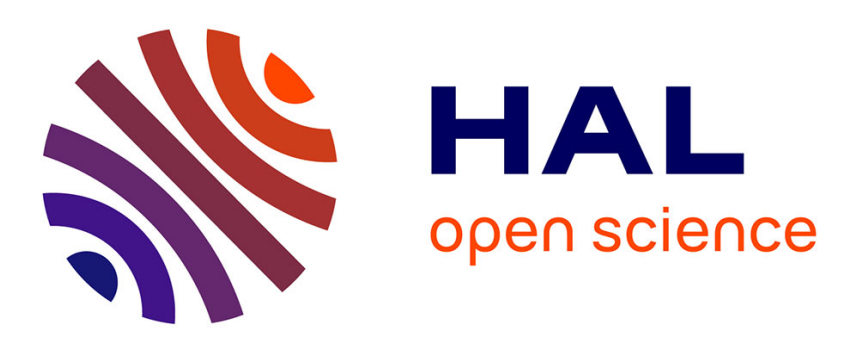

\title{
Détermination des énergies d'échange entre les ions manganèse de plusieurs complexes manganèse-amine luminescents
}

J. Mimault, J. Delafond, A. Junqua, J. Hervé

\section{- To cite this version:}

J. Mimault, J. Delafond, A. Junqua, J. Hervé. Détermination des énergies d'échange entre les ions manganèse de plusieurs complexes manganèse-amine luminescents. Revue de Physique Appliquée, 1971, 6 (4), pp.441-444. 10.1051/rphysap:0197100604044100 . jpa-00243568

\section{HAL Id: jpa-00243568 \\ https://hal.science/jpa-00243568}

Submitted on 1 Jan 1971

HAL is a multi-disciplinary open access archive for the deposit and dissemination of scientific research documents, whether they are published or not. The documents may come from teaching and research institutions in France or abroad, or from public or private research centers.
L'archive ouverte pluridisciplinaire HAL, est destinée au dépôt et à la diffusion de documents scientifiques de niveau recherche, publiés ou non, émanant des établissements d'enseignement et de recherche français ou étrangers, des laboratoires publics ou privés. 


\title{
DÉTERMINATION DES ÉNERGIES D'ÉCHANGE ENTRE LES IONS MANGANĖSE DE PLUSIEURS COMPLEXES MANGANÈSE-AMINE LUMINESCENTS
}

\author{
J. MimaulT, J. DELAFOND, A. JUNQUA \\ Laboratoire de Métallurgie Physique, Faculté de Sciences de Poitiers, France \\ et J. HERVÉ \\ Laboratoire d'électronique, Université de Provence St-Jérôme Marseille, France
}

(Reçu le 2 juillet 1971)

\begin{abstract}
Résumé. - Nous présentons, pour divers complexes manganèse-amine de luminescence différente, les courbes expérimentales de susceptibilité magnétique en fonction de la température dans la gamme $10^{\circ} \mathrm{K}-300^{\circ} \mathrm{K}$. Les mesures ont été effectuées par la méthode de Faraday.

L'interprétation de ces courbes dans leur région paramagnétique, au moyen de la théorie de Kramers-Opechowski, nous donne la valeur de l'énergie d'échange entre les ions magnétiques de chaque échantillon étudié.
\end{abstract}

\begin{abstract}
We report experimental results on the magnetic susceptibility of several manganese-amine complexes, the luminescence of which are different. Measurements were made by the Faraday method between $10^{\circ} \mathrm{K}$ and $300{ }^{\circ} \mathrm{K}$.

The interpretation of experiments by means of the Kramers-Opechowski theory gives the magnitude of the exchange interaction between magnetic ions in each sample.
\end{abstract}

I. Introduction. - Slivitch [1] avait découvert dès 1957 que les composés complexes résultant de l'action du chlorhydrate d'une amine sur le chlorure de manganèse étaient des corps luminescents.

Payen de la Garanderie [2] a étudié le spectre de tels complexes, en fonction de la nature et du nombre de ligands.

Leurs propriétés magnétiques ont déjà été étudiées par Gayda [3] par résonance paramagnétique électronique : il avait pu constater que l'énergie d'échange entre ions métalliques variait notablement suivant l'amine utilisée; en outre, il avait mis en évidence une corrélation entre la grandeur de cette énergie et l'extinction de la luminescence.

Nous décrivons ici des mesures statiques de susceptibilité magnétique réalisées entre $10^{\circ} \mathrm{K}$ et $300^{\circ} \mathrm{K}$. Leur interprétation nous a permis de confirmer la valeur $S=5 / 2$ du spin de l'ion manganèse, de connaître le comportement magnétique de ces échantillons et de donner la valeur des énergies d'échange correspondantes.

II. Résultats expérimentaux. - a) APPAReILLAGE. Nous utilisons la méthode de Faraday. Les échantillons sont réduits en poudre et placés dans une nacelle en or; le champ magnétique est stabilisé à $10^{-6}$, les forces sont mesurées à l'aide d'une électrobalance
Cahn sensible à $0,1 \mu \mathrm{g}$ dans la gamme utilisée. Les susceptibilités ont été calculées en prenant le sel de Mohr comme échantillon de référence :

$\chi_{\text {sel de Mohr }}=32,6 \times 10^{-6}$ u. e. m. c. g. s./g à $17,2^{\circ} \mathrm{C}[4]$.

Un cryostat à circulation (Air Liquide) et une régulation (Meric) permettent d'obtenir toute température entre $10^{\circ} \mathrm{K}$ et l'ambiante avec une stabilité de $0,05^{\circ} \mathrm{K}$. La détection en température est réalisée au moyen d'une sonde d'arséniure de gallium placée à $5 \mathrm{~mm}$ de l'échantillon, et préalablement étalonnée en position de mesure. L'échange thermique entre la source froide et le corps étudié est assuré par de l'hélium gazeux.

b) CoRps ÉTUdiés. - Les complexes ont été fabriqués au laboratoire; nous avons choisi de présenter les résultats correspondants à quatre d'entre eux, parce qu'ils possèdent des propriétés de luminescence différentes.

Ce sont :

$$
\begin{aligned}
& \mathrm{MnCl}_{2}, 2 \mathrm{CH}_{3} \mathrm{NH}_{2}, \mathrm{HCl} \\
& \mathrm{MnCl}_{2}, 2 \mathrm{C}_{2} \mathrm{H}_{5} \mathrm{NH}_{2}, \mathrm{HCl} \\
& \mathrm{MnCl}_{2},\left(\mathrm{CH}_{3}\right)_{2} \mathrm{NH}, \mathrm{HCl} \\
& \mathrm{MnCl}_{2}, \mathrm{C}_{5} \mathrm{H}_{5} \mathrm{~N}, \mathrm{HCl}
\end{aligned}
$$


c) Courbes $\chi=f(T)$ (Fig. 1).

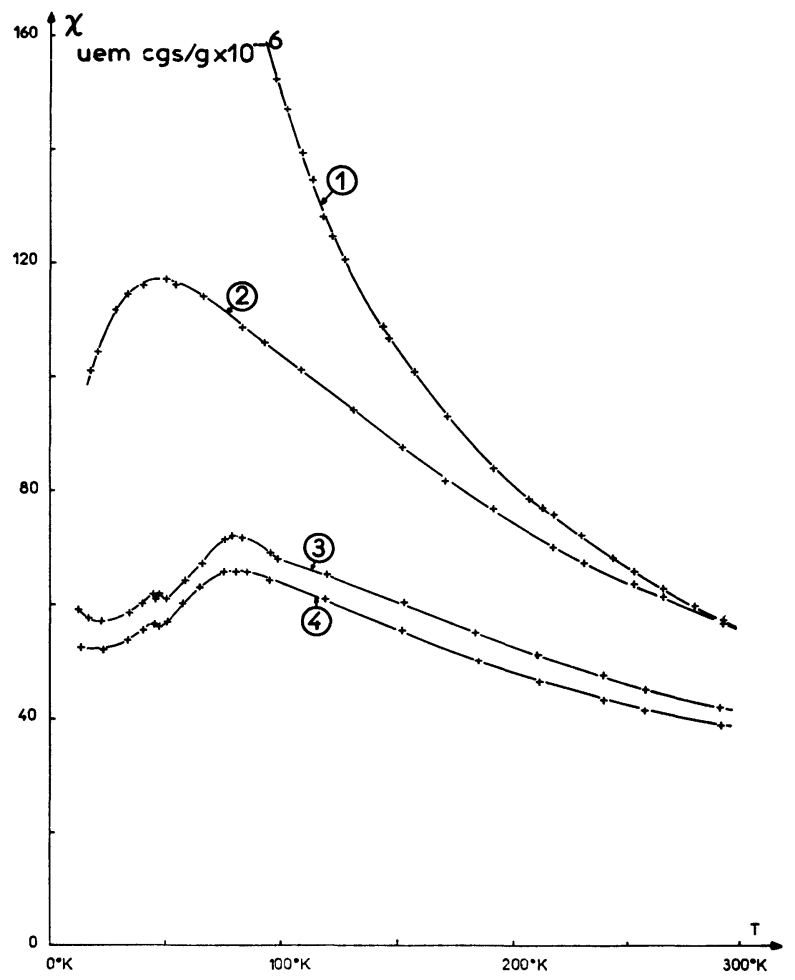

FIG. 1. - Susceptibilité magnétique des complexes :
1. $\mathrm{MnCl}_{2},\left[\mathrm{C}_{5} \mathrm{H}_{5} \mathrm{~N}, \mathrm{HCl}\right]$
2. $\mathrm{MnCl}_{2},\left[\left(\mathrm{CH}_{3}\right)_{2} \mathrm{NH}, \mathrm{HCl}\right]$
3. $\mathrm{MnCl}_{2}, 2\left[\mathrm{CH}_{3} \mathrm{NH}_{2}, \mathrm{HCl}\right]$
4. $\mathrm{MnCl}_{2}, 2\left[\mathrm{C}_{2} \mathrm{H}_{5} \mathrm{NH}_{2}, \mathrm{HCl}\right]$

Nous remarquons le comportement antiferromagnétique de ces corps (présence d'un point de Néel, susceptibilité de l'ordre de $10^{-4}$ u. e. m. c. g. s./g). Ces résultats démontrent l'existence d'une interaction d'échange négative entre les ions $\mathrm{Mn}^{2+}$.

Nous ne discuterons pas quantitativement la région de magnétisme ordonné. (On notera cependant la forme particulière des variations de $\chi$ pour les échantillons 3 et 4.) Par contre, l'interprétation de la région paramagnétique nous permettra de chiffrer l'intégrale d'échange.

III. Interprétation théorique des résultats obtenus. a) EXPRESSION GÉNÉRALE DE LA SUSCEPTIBILITÉ. — La théorie employée est celle donnée par Kramers et Opechowski [5]. Elle tient compte des interactions isotropes entre premiers voisins. L'hamiltonien de Heisenberg s'écrit :

$$
\mathscr{H}_{\mathrm{P}}=-2 J \sum_{<i, j>} \mathbf{S}_{i} \mathbf{S}_{j}+g \beta \mathbf{H}_{0} \sum_{k} \mathbf{S}_{k}
$$

$J$ : intégrale d'échange entre deux ions premiers voisins.

$H_{0}$ : champ magnétique extérieur appliqué.

$\sum_{\langle i, j\rangle}$ : somme effectuée sur toutes les paires $(i, j)$ de premiers voisins appartenant à l'ensemble d'ions.
La somme $\sum_{k}$ porte sur tous les ions considérés.

$g:$ facteur de Landé. (Dans le cas de l'ion $\mathrm{Mn}^{2+}$ : $g \simeq 2$.)

La susceptibilité paramagnétique $\chi$ est calculée à l'aide de la formule :

$$
\chi=\left[\frac{\partial^{2}}{\partial H^{2}}(k T \ln Z)\right]_{H=0}
$$

où $Z$ est la fonction de partition.

Plusieurs auteurs [6], [7] ont donné des méthodes de résolution numérique; ils ont exprimé le résultat sous la forme :

$$
\chi(t)=\chi_{0}(t) \sum_{n=0}^{\infty} \frac{a_{n}}{t^{n}}
$$

avec

$$
\left\{\begin{array}{c}
t=\frac{k T}{J} \\
\chi_{0}(t)=\frac{k}{J} \frac{C}{t} .
\end{array}\right.
$$

$C$ : constante de Curie.

Rushbrooke et Wood [6] ont donné des formules pour les six premiers coefficients dans le cas d'un spin quelconque. Stanley et Kaplan [7] ont calculé les neuf premiers coefficients dans l'approximation classique du spin ; leurs résultats sont pratiquement identiques à ceux de Rushbrooke et Wood pour $S=5 / 2$. Tous ces résultats sont valables pour $k T /|J| \gg 1$.

$b$ ) CALCUl DeS COEFFiCIENTS $a_{n}$ POUR LeS CORPS ÉTUDIÉs. - Les coefficients $a_{n}$ dépendent de la valeur $\mathrm{du}$ spin et de certaines caractéristiques du réseau. De récents travaux [8] permettent d'affirmer que la structure du complexe $-\mathrm{MnCl}_{2},\left[\left(\mathrm{C}_{5} \mathrm{H}_{5} \mathrm{~N}\right), \mathrm{HCl}\right]$ est monoclinique proche du quadratique. Nous faisons l'hypothèse qu'il en est de même pour les aatres complexes étudiés; les différents coefficients $a_{n}$ sont alors les suivants :

Coefficients $\{A\}$ calculés

d'après les formules de Rushbrooke et Wood

Coefficients $\{$ B $\}$ calculés par Stanley et Kaplan

$$
\begin{aligned}
& a_{0}=1 \\
& a_{1}=2,33333 \times 10 \\
& a_{2}=3,96588 \times 10^{2} \\
& a_{3}=6,21104 \times 10^{3} \\
& a_{4}=8,73955 \times 10^{4} \\
& a_{5}=11,51314 \times 10^{5} \\
& a_{6}=14,50044 \times 10^{6}
\end{aligned}
$$

$$
\begin{aligned}
& a_{0}=1 \\
& a_{1}=2,3333 \times 10 \\
& a_{2}=4,0833 \times 10^{2} \\
& a_{3}=6,6694 \times 10^{3} \\
& a_{4}=9,91151 \times 10^{4} \\
& a_{5}=13,9718 \times 10^{5} \\
& a_{6}=18,9448 \times 10^{6} \\
& a_{7}=24,7545 \times 10^{7} \\
& a_{8}=31,4964 \times 10^{8} \\
& a_{9}=38,9341 \times 10^{9}
\end{aligned}
$$


c) Prolongement analytique. - Les séries (1) divergent pour les températures inférieures à

$$
T_{\mathrm{R}}=\frac{J}{k \lim \left(a_{n}\right)^{-1 / n}} .
$$

La valeur de $T_{\mathbf{R}}$ peut être assez grande pour être placée dans la région paramagnétique désordonnée.

Pour les corps antiferromagnétiques, Daniellian et Stevens [9] ont trouvé un prolongement analytique de la série, valable pour $T<T_{\mathrm{R}}$. Ce prolongement est de la forme :

$$
\chi(|t|)=\frac{k C}{|J|[|t|+\theta]} \sum_{n=0}^{\infty} \frac{(-1) d_{n}}{(|t|+\theta)^{n}}
$$

avec :

$$
d_{n}=\sum_{r=0}^{n}(-1)^{r} a_{n-r} C_{n}^{r} \theta^{n}
$$

Daniellian et Stevens montrent que la valeur de $\chi(|t|)$ donnée par la formule (2) est indépendante de $\theta$ pourvu que l'on ait $\theta>\frac{1}{2} k T_{\mathrm{R}} / J$.

Comme nous ne disposons que d'un nombre fini $N$ de termes $a_{n}$, on est amené à limiter la série au $n$-ième et $\chi(|t|)$ devient alors fonction de $\theta$.

Daniellian et Stevens ont montré que l'erreur ainsi commise devient minimum en choisissant une valeur de $\theta$ racine du coefficient $d_{N}$. Ces deux conditions nous conduisent à prendre $\theta=100$ pour les coefficients du calcul de Rushbrooke et Wood, et $\theta=120$ pour ceux du calcul de Stanley et Kaplan.

Les valeurs des coefficients $d_{n}$ sont alors les suivantes :

Théorie de Rushbrooke et Wood

(coefficients $\{\mathrm{C}\}$ )

$d_{1}=-0, \overline{766} 66 \times 10^{2}$

$d_{2}=0,57299 \times 10^{4}$

$d_{3}=-0,41277 \times 10^{6}$

$d_{4}=0,26065 \times 10^{8}$

$d_{5}=-0,17208 \times 10^{10}$

$d_{6}=0$
Théorie de Stanley et Kaplan

(coefficients $\{\mathrm{D}\}$ ) $d_{1}=-0, \overline{966} 66 \times 10^{2}$

$d_{2}=0,92482 \times 10^{4}$

$d_{3}=-0,86051 \times 10^{6}$

$d_{4}=0,78258 \times 10^{8}$

$d_{5}=-0,68448 \times 10^{10}$

$d_{6}=0,52234 \times 10^{12}$

$d_{7}=-0,41163 \times 10^{14}$

$d_{8}=0,22690 \times 10^{16}$

$d_{9}=0$ d) Calcul DE L'ÉNergie D'éChange. - Sur la figure 2 nous représentons, d'une part les courbes théoriques $\chi(|t|) / \chi_{0}(|t|)$ calculées suivant les différentes théories, d'autre part les résultats expérimentaux sous la forme $\chi(T) / \chi_{0}(T), \chi_{0}(T)$ étant calculé au moyen de la loi de Curie. La correspondance entre les points expérimentaux et théoriques de même ordonnée fournit de suite le rapport :

$$
\frac{T}{|t|}=\frac{|J|}{k} \text {. }
$$

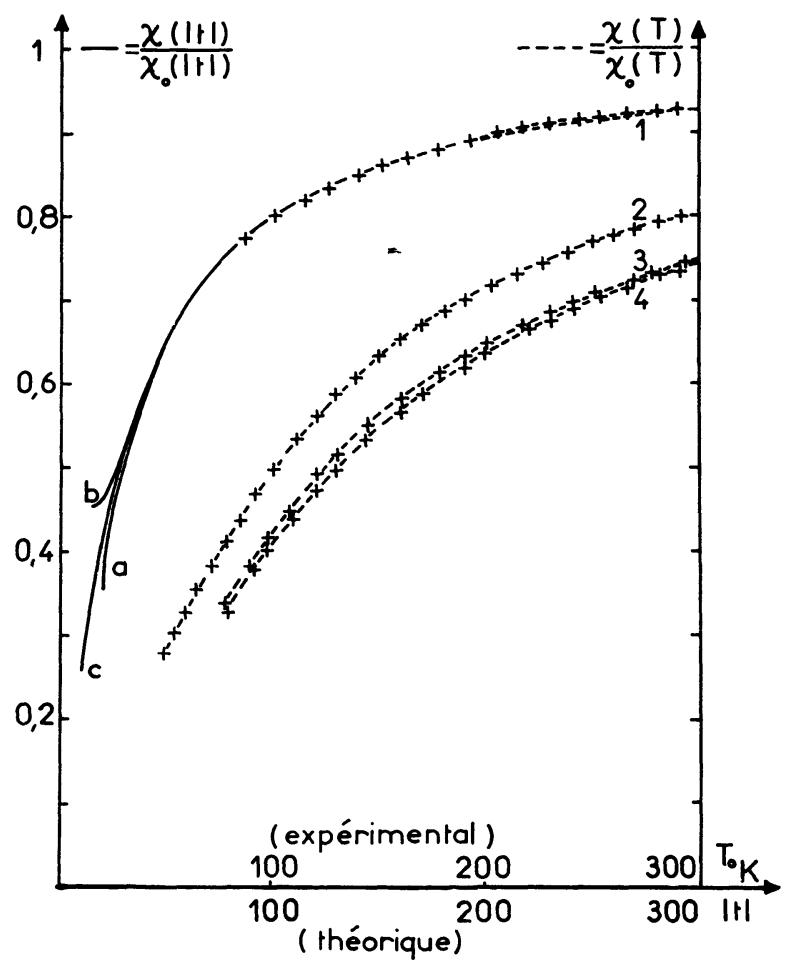

Fig. 2. - — : Courbes théoriques : a) Courbe tracée avec les coefficients $\{\mathrm{A}\}$ de Stanley et Kaplan. $b$ ) Courbe tracée avec les coefficients $\{$ B $\}$ de Rushbrooke et Wood. c) Courbe due au prolongement analytique coefficients $\{C\}$ et $\{D\}$. (Les 2 séries donnent le même résultat.) - - - - - : Courbes expérimentales des divers échantillons (mêmes notations que pour la figure 1).

Pour rester dans le domaine de l'approximation des hautes températures, nous ne considérerons que

\begin{tabular}{|c|c|c|c|c|c|c|c|c|c|}
\hline$\chi(|t|) / \chi_{0}(|t|)=\chi(T) / \chi_{0}(T)$ & & 0,60 & 0,65 & 0,70 & 0,75 & 0,80 & 0,85 & 0,90 & 0,925 \\
\hline$|t|$ & & 40,5 & 49 & 60,5 & 75 & 99 & 137,5 & 210 & 287 \\
\hline $\mathrm{MnCl}_{2}, 2\left[\mathrm{CH}_{3} \mathrm{NH}_{2}, \mathrm{HCl}\right]$ & $\begin{array}{l}T\left({ }^{\circ} \mathrm{K}\right) \\
J / k\left({ }^{\circ} \mathrm{K}\right)\end{array}$ & $\begin{array}{c}176 \\
-4,34\end{array}$ & $\begin{array}{c}208 \\
-4,21\end{array}$ & $\begin{array}{c}\overline{249} \\
-4,15\end{array}$ & & & & & \\
\hline $\mathrm{MnCl}_{2}, 2\left[\mathrm{C}_{2} \mathrm{H}_{5} \mathrm{NH}_{2}, \mathrm{HCl}\right]$ & $\begin{array}{l}T\left({ }^{\circ} \mathrm{K}\right) \\
J / k\left({ }^{\circ} \mathrm{K}\right)\end{array}$ & $\begin{array}{c}170 \\
-4,2\end{array}$ & $\begin{array}{r}202 \\
-4,1\end{array}$ & $\begin{array}{c}245 \\
-4,05\end{array}$ & $\begin{array}{c}300 \\
-4\end{array}$ & & & & \\
\hline $\mathrm{MnCl}_{2},\left[\mathrm{CH}_{3} \mathrm{NH}_{2}, \mathrm{HCl}\right)$ & $\begin{array}{l}T\left({ }^{\circ} \mathrm{K}\right) \\
J / k\left({ }^{\circ} \mathrm{K}\right)\end{array}$ & $\begin{array}{r}136 \\
-3,3\end{array}$ & $\begin{array}{r}158,5 \\
-3,2\end{array}$ & $\begin{array}{c}189 \\
-3,1\end{array}$ & $\begin{array}{c}230 \\
-3,05\end{array}$ & $\begin{array}{l}297 \\
-3\end{array}$ & & & \\
\hline $\mathrm{MnCl}_{2},\left[\mathrm{C}_{5} \mathrm{H}_{5} \mathrm{~N}, \mathrm{HCl}\right]$ & $\begin{array}{l}T\left({ }^{\circ} \mathrm{K}\right) \\
J / k\left({ }^{\circ} \mathrm{K}\right)\end{array}$ & & & & & $\begin{array}{r}99 \\
-1\end{array}$ & $\begin{array}{c}138 \\
-1\end{array}$ & $\begin{array}{c}211 \\
-0,97\end{array}$ & $\begin{array}{c}282 \\
-0,98\end{array}$ \\
\hline
\end{tabular}
les résultats obtenus pour $t>40$. Ceux-ci sont groupés dans le tableau I.

TABLEAU I 
Nous remarquons que pour $\mathrm{MnCl}_{2},\left[\mathrm{C}_{5} \mathrm{H}_{5} \mathrm{~N}, \mathrm{HCl}\right]$, les courbes expérimentale et théorique sont pratiquement confondues.

Dans les autres cas, les résultats ont tendance à diverger un peu.

Ceci peut être attribué au fait que nous n'avons pas tenu compte du phénomène d'ordre à courte distance dont les effets ne disparaissent qu'à haute température.

Conclusion. - La comparaison avec l'intensité émise par ces complexes lorsqu'ils sont placés dans des conditions identiques d'excitation et de détection confirme la théorie de Dexter [10] attribuant un rôle extincteur de luminescence aux interactions d'échange.

Celles-ci sont négatives et les résultats montrent que ces interactions, en corrélation avec la grosseur des amines, restent faibles. Il serait donc possible de traiter l'ion manganèse dans un modèle de champ cristallin.

Pour confirmer cette hypothèse, il sera utile de relier l'intégrale d'échange aux caractéristiques du spectre optique donné par les mesures de luminescence.

\section{Bibliographie}

[1] Slivitch (S.), C. R. Acad. Sci., Paris, 1957, 245, 2047.

[2] Payen de la Garanderie, Annales de Physique, 1964, 9, 649, C. R. Acad. Sci., Paris, 1962, 254, 2739.

[3] Gayda (J. P.), C. R. Acad. Sci., Paris, 1967, 265, 561.

[4] Bates (L. F.), Modern magnetism, Cambridge University Press, Cambridge, 1951.

[5] Opechowski, Physica, 1937, 4, $181 ; 1938,6,1112$.

[6] Rushbrooke (G. S.) et Wood (P. S.), Proceeding on physical society, 1955, A 68, $1161 ; 1955, \mathbf{A} 70$, 765 ; Mol. Phys., 1958, 1, 250.
[7] Stanley (H. E.), Kaplan (T. A.), Phys. Rev., 1967, 2, 158 ; 1967, 2, 158 ; Phys. Rev. Letters, 1966, 981, 16, 22, J. appl. Phys., 1965, 1129, 36, 3.

[8] RoBert (Poitiers) (non publié).

[9] Daniellian (A.) et Stevens (K. W. H.), Proceeding on physical Society of London, 1961, 77, 116; 1961, 77, 124.

[10] Dexter (D. L.), J. Chem. Phys., 1953, 21, 836; 1954, 22, 1063. 